\title{
A WPAN Platform Design in Mobile Phone Considering Application Development and Usability
}

\author{
I.-H. Kim, G.-M. Jeong, E.-C. Park, K.-D. Chung
}

\author{
In-Hwan Kim \\ Mobile Device Development Team \\ SK Telecom, Seoul, Korea \\ E-mail: inhwan.kim@sk.com

\section{Gu-Min Jeong} \\ School of Electrical Engineering \\ Kookmin University, Seoul, Korea \\ E-mail: gm1004@kookmin.ac.kr

\section{Eun-Chan Park} \\ Department of Information and \\ Communication Engineering \\ Dongguk University-Seoul, Korea \\ E-mail: ecpark@dongguk.edu
}

\section{Ki-Dong Chung}

School of Computer Science and Engineering

Pusan National University, Pusan, Korea

E-mail: kdchung@pusan.ac.kr

\begin{abstract}
In this paper, we propose a WPAN (Wireless Personal Area Network) platform for converged network services integrating a cellular network and a WPAN. We mainly focus on an easy-to-develop and easy-to-use WPAN platform for wireless communication services using both networks. The proposed WPAN platform consists of a WPAN handset platform, a WPAN connection scheme, and a WPAN server platform. The WPAN handset platform provides abstract WPAN API (Application Programming Interface) set and application management module. Using the WPAN connection scheme, the user can enjoy converged network services in a convenient way. The WPAN server platform manages the overall services and digital devices that are connected to the handset. Compared to the existing WPAN related platform, we consider the need for the integrated service components allowing for the development of WPAN applications in the handset and their convenience for the user. Also, illustrative services and devices are implemented using the proposed method, which show the applicability of the proposed WPAN platform.

Keywords: WPAN, Converged Network, Platform, API Abstraction, Connection Scheme, Server Structure, Mobile Game
\end{abstract}

\section{Introduction}

Mobile phones are becoming essential devices thanks to their portability and mobility. Numerous new technologies are being merged into mobile handsets and various new services are provided based on cellular networks, leading to the development of ubiquitous services [1].

Recently, wireless network technologies such as Mobile WiMAX (Worldwide Interoperability for Microwave Access), WPAN, and WLAN (Wireless Local Area Network) have become alternatives to high rate data services. Although each technology has its own benefits, enhanced 
services cannot be developed without a cellular network. With this in mind, various studies have been conducted on the interoperability and integration for the heterogeneous networks [2]-[9].

Among these, WLAN and WPAN are generally adopted in mobile phones. Although $3 \mathrm{G}$ and WLAN interworking makes WLAN popular in smart phones, WPAN [10]-[12], which is widely used in mobile phones, also has its own advantages such as convenient device connection and reduced power consumption for mobile phones. Especially, Bluetooth [10] is used in mobile phone for the headset and data transmission. However, since the main objective of the WPAN is to connect devices without cables, the usage of WPAN in mobile phone is somewhat restrictive when it comes to providing ubiquitous network services [2], [13]. For example, Bluetooth is used only for the headset, the connection to a PC, or the connection to other handsets. Likewise, the use of the WPAN in mobile phone appears to be independent of the cellular network. This has been a major drawback of the application of WPAN to mobile phones.

Up to now, there have been many studies on the integration of WPAN and cellular networks and the convenience of such integration for the user. With JSR (Java Specification Request)-82 [14], Bluetooth APIs are provided in the J2ME (Java 2 Platform, Micro Edition) environment, which makes it possible to utilize both Bluetooth and a cellular network. The Gaia platform [15] supports a middleware for ad-hoc pervasive computing. Also, CTIA (Cellular Telephone Industries Association) provides a certification and test rules for the interoperability of Bluetooth devices in order to enhance their usability [16]-[17].

Considering these points discussed herein, we can think that for the converged network services, there must be consideration for the APIs in mobile phone, the user scenario, server structure and application management.

In this paper, we propose a WPAN platform that integrates a cellular network and a WPAN, in order to provide ubiquitous network services and implement converged network services. We primarily consider the development of services which utilize both networks fully. Compared to the existing WPAN integrations, we focus on the platform environment and user applicability of the wireless communication services being developed. We summarize the concept of the converged network service and propose a WPAN handset platform, a WPAN connection scheme, and a WPAN server platform in this paper. For the design of the WPAN handset platform, we investigate various use cases of service scenarios and integrate the cellular network and the WPAN in mobile handsets. The WPAN server platform takes control of the services and devices connected to the handset. Also, for the convenience of the user, a WPAN connection scheme is presented.

Although the platform is implemented with Bluetooth at present, the overall architecture is designed to take into consideration its extension to other WPAN technologies, and the implementation of ZigBee and UWB (Ultra-Wide Band) is currently underway.

To show the validity of the proposed platform, we present various illustrative implementation and service examples such as a PC to phone application, phone to phone game, Voice Terminal, PMP (Portable Media Player) and LBS (Location Based Service).

The remainder of this paper is organized as follows. In Section II, related work is briefly introduced. In Section III, we summarize the basic concept of the converged network services and the proposed WPAN platform. In Sections IV, V and VI, we present the WPAN handset platform, the WPAN connection scheme, and the WPAN server platform, respectively. In Section VII, performance evaluation is presented considering number of API calls. In Section IIX, service implementations are described and the conclusion follows in Section IX. 


\section{Related Works}

Since WPAN is widely used in mobile phones, many studies have been conducted on integration of WPAN and various standards have been established. Also, many researches have been performed on the implementation of ubiquitous services.

Until now, the integration of WPAN in mobile phones has generally focused on the WPAN functions in the mobile phone. However, little consideration has been given to the development of converged network services and the convenience of the user.

JSR-82 (JavaTM APIs for Bluetooth) [14] is a standard of JCP (Java Community Process), which provides Bluetooth APIs for the J2ME environment. There are standard APIs for the various Bluetooth profiles. Using JSR-82, WPAN functions can be supported in handsets. JSR82 supports only Bluetooth APIs and there still remain other platform components for converged network services.

Recently, CTIA makes a certification program for Bluetooth, viz. the BCCP (Bluetooth Compatibility Certification Program). Bluetooth devices made by different companies provide different user scenarios and this may cause inconvenience to the user. Considering these points, CTIA presents the BCCP with consideration given to the end-user perspective [16]-[17].

To provide ubiquitous services, many platform structures have been suggested [18]. Among them, Gaia [15] provides a middleware for ad-hoc pervasive computing. The structure of Mobile Gaia supports various mediums such as Bluetooth, IrDA, Wi-Fi, Ethernet, etc. and provides core services such as discovery and cluster management services, event services, location services, context services and security services.

Also, PNM (Personal Network Management) [19]-[20] deals with the composition of private networks for UMTS or GSM networks and the control of the UE (User Equipment). PNM is a home network-based application provides for the home network-based management of Personal Networks (PN) consisting of multiple devices belonging to a single user, as described in 3GPP TS 22.259. Basically, PNM describes the protocols required for the composition of private networks and user equipment.

As described in related works, in order to provide WPAN services and ubiquitous services efficiently in mobile phones, we should consider the easy development and the easy use of WPAN applications. Considering these points and related works, a WPAN platform is designed and implemented in this study.

\section{Basic Concept of Converged Network Services}

In this section, we present a basic concept of the proposed WPAN platform and the WPAN platform-based services.

WPAN technologies are mainly used for the connection between the mobile handset and other devices. They are adopted only for data transmission among devices or for headset functions. However, if we integrate the cellular network and the WPAN effectively, various converged network services can be developed easily.

Table 1 shows service examples of the integration of WPAN and the cellular network. As shown in Table 1, handsets can connect to handsets, application devices, and access points. Various ubiquitous services can be provided using the cellular network and the WPAN.

This paper proposes a WPAN platform which enables the service providers to develop the services listed in Table 1 more easily. Considering the service development of services, we aim to design a WPAN platform that integrates the cellular network and the WPAN effectively.

In fact, the services in Table 1 could be developed without the proposed WPAN platform. However, they can be developed more easily if the proposed WPAN platform is applied and the 
Table 1: The classification of WPAN services

\begin{tabular}{|c|c|}
\hline Service Type & Example \\
\hline Handset to Handset Services & $\begin{array}{c}\text { Community Service, Contents Distribution, } \\
\text { Handset to Handset game, etc. }\end{array}$ \\
\hline Handset to Application Device Services & $\begin{array}{r}\text { Dial-Up Networking, SMS Forwarding, Phonebook Sync, } \\
\text { Contents Download for Application Device }\end{array}$ \\
\hline Handset to Access Point Services & $\begin{array}{c}\text { Zone Based Services such as Location-based Service, } \\
\text { Personalized Service, D-Home, Home Security, etc. }\end{array}$ \\
\hline
\end{tabular}

service scenario can be more practical.

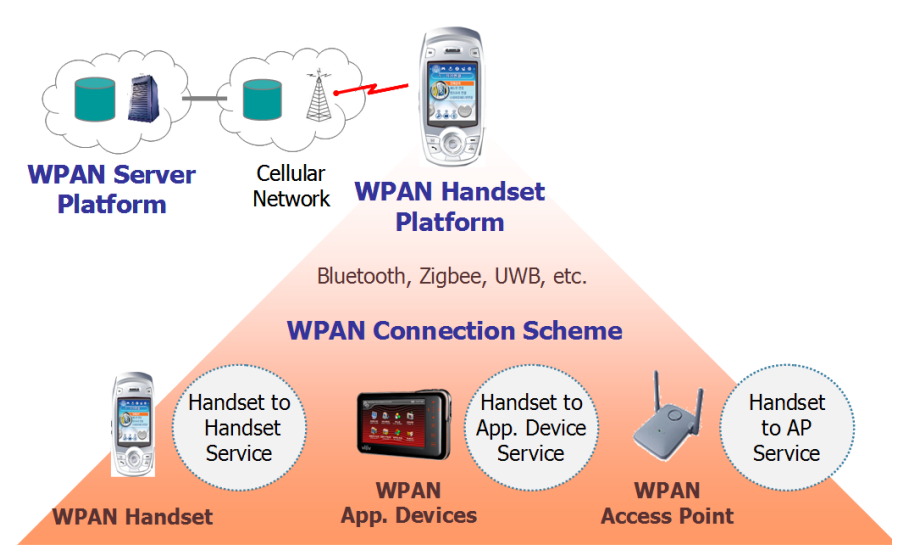

Figure 1: Overview of various converged network services

Figure 1 shows the basic concept of the proposed WPAN platform and the service flow. The proposed platform consists of the WPAN handset platform, the WPAN server platform, and the WPAN connection scheme. The WPAN handset platform provides abstract WPAN APIs and an application management module that enables service developers to create WPAN services more easily. The WPAN connection scheme offers a convenient user scenario for WPAN services. The converged network services and service scenarios may be unfamiliar to the user. Using this scheme, we can make the service scenario easy to use. The WPAN server platform manages the devices connected to the handset provides the handset with appropriate services. Also, provisioning and downloads are handled in this platform.

\section{WPAN HANDSET PLATFORM}

This section deals with the WPAN handset platform. We design abstract APIs and an application management module in this platform.

\subsection{Basic structure of the WPAN handset platform}

For the developers who are not familiar with WPAN technology, we aim to provide an easy development environment using this platform. Considering this objective, the major characteristics of the proposed platform are as follows: 
- We provide both cellular network APIs and WPAN APIs for the developers.

- The WPAN APIs are abstracted for the easy development of WPAN applications.

- Though the current implementation is for Bluetooth, the platform design considers its extension to ZigBee and UWB.

- Different from other applications, WPAN applications should be executed without any user input. We provide the application management module and PAN Agent for this purpose.

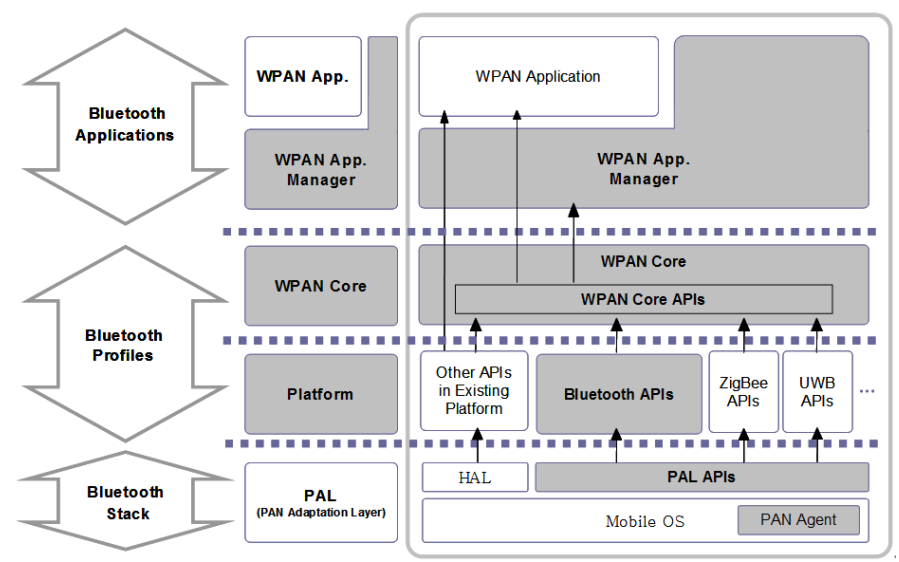

Figure 2: WPAN handset platform architecture

Figure 2 shows the WPAN handset platform architecture. In order to implement abstract APIs, we design a layered structure. The gray areas in Figure 2 are the platform components of the WPAN handset platform. The WPAN APIs have three layers, which are the PAL Layer, Platform Layer, and WPAN Core Layer. Also, for the management of WPAN applications and their connections, the WPAN Application Manager (WAM) and the PAN Agent are provided. The detailed descriptions of the each abstraction layer are as follows:

- PAL (PAN Adaptation Layer) is a HAL (Hardware Adaptation Layer) related to WPAN technologies and implemented by the manufacturer.

- In the Platform Layer, APIs for each WPAN technology (e.g., Bluetooth, ZigBee, UWB, etc.) are implemented using the PAL Layer. In order to use the APIs in the Platform Layer, developers must know each WPAN technology in detail. Besides the WPAN APIs, the Platform Layer supports numerous API sets such as UI, memory, and process.

- The WPAN Core Layer provides the encapsulated WPAN Core APIs, which are independent of the WPAN technologies. Using WPAN Core APIs, services can be implemented without any specific knowledge of each WPAN technology. Also, the applications are independent of the WPAN physical layer.

For the management of the WPAN applications and connections, the WPAN platform provides the WAM (WPAN Application Manager) and the PAN Agent.

- The WAM handles the WPAN applications, user interface, and WPAN events that are sent to the handset. The WAM can be implemented using the Platform Layer and the WPAN Core API. 
- The PAN Agent is a kind of daemon process and always runs in background mode. Regardless of the state of the handset, the PAN agent receives WPAN events and delivers them to the WAM. Therefore, though the handset is in an idle state, it can receive and send WPAN events to the WAM.

For developers who are familiar with each WPAN technology, PAL APIs can be used directly. In this case, it is possible to access each profile directly.

\subsection{Design of Abstract WPAN APIs}

Table 2: Abstraction Example from the PAL to the WPAN Core APIfor the Connection Function

\begin{tabular}{|c|c|c|}
\hline Layer & Description & Prototype \\
\hline WPAN Core API & Connect to device & M_Int32 WPAN_Connect(M_Int32 fd, Callback cb); \\
\hline WPAN API & $\begin{array}{c}\text { Get fd for BT } \\
\text { SPP connection }\end{array}$ & $\begin{array}{c}\text { M_Int32 BT_Connect(M_Int32 spp_fd,MC_BluetoothSDPInfo } \\
\text { *btSDPInfo, SVCCONNECTCB cnncb); }\end{array}$ \\
\hline PAL API & $\begin{array}{c}\text { Get fd for BT } \\
\text { SPP connection }\end{array}$ & $\begin{array}{c}\text { Typedef M_Int32 }\left({ }^{*} \text { DEVCONTROLFUN) }\right. \\
\text { (M_UInt16 devnum, M_char *cmd, void *param1, void *param2); }\end{array}$ \\
\hline
\end{tabular}

Table 2 shows the abstraction step of APIs for Bluetooth, where the WPAN_connect() API is presented. The PAL Layer is an implementation of a device driver level. In the Platform Layer, there is a Bluetooth-level API. Finally, in the WPAN Core Layer, WPAN_Connect() is provided. Developers can get the fd (file descriptor) for the communication and then use the WPAN_Connect() API. Therefore, without specific knowledge of the Bluetooth SDP and SPP, one can develop WPAN applications easily.

Table 3: WPAN Core APIs

\begin{tabular}{|c|c|}
\hline APIs & Descriptions \\
\hline WPAN_ONOFF() & $\begin{array}{l}\text { Turn on/off the hardware module for the WPAN } \\
\text { (Bluetooth, ZigBee, UWB, etc.) in the WPAN handset. }\end{array}$ \\
\hline WPAN_Create() & $\begin{array}{l}\text { In the server mode of WPAN handset, get the fd after making } \\
\text { a serial port channel, and then remain in the listen state. }\end{array}$ \\
\hline WPAN_GetPairedList() & Get the list of devices that were connected before. \\
\hline WPAN_Connect() & $\begin{array}{l}\text { In the client mode of the WPAN handset, connect to the server } \\
\text { and get the fd for the connected serial port channel. }\end{array}$ \\
\hline WPAN_Disconnect() & $\begin{array}{l}\text { Disconnect the serial port connection } \\
\text { between the server and the client. }\end{array}$ \\
\hline WPAN_Remove () & $\begin{array}{l}\text { In the server mode of the WPAN handset, } \\
\text { release the listen state of the serial port channel }\end{array}$ \\
\hline WPAN_spClose() & Close the connected serial port channel. \\
\hline WPAN_SendData() & Send data to the connected serial port channel. \\
\hline WPAN_ecvData () & Receive data from the connected serial port channel. \\
\hline WPAN_GetLocalInfo() & $\begin{array}{l}\text { Get the information such as device name, } \\
\text { BD_ADDR of the WPAN handset. }\end{array}$ \\
\hline WPAN_GetLibVersion() & Get the version information of the WPAN Core Library. \\
\hline
\end{tabular}

Table 3 shows the list of WPAN Core APIs. As shown in Table 3, WPAN Core APIs are independent of WPAN technologies.3 The proposed WPAN handset platform is implemented 
only for Bluetooth. However, the applications developed with the proposed platform can be easily adapted for other WPAN technologies, as shown in Figure 3. Figure 3 shows an example of a game application. Since the application is independent of the physical layer, it is possible to re-use the source code of the applications and run them regardless of the WPAN physical layer.

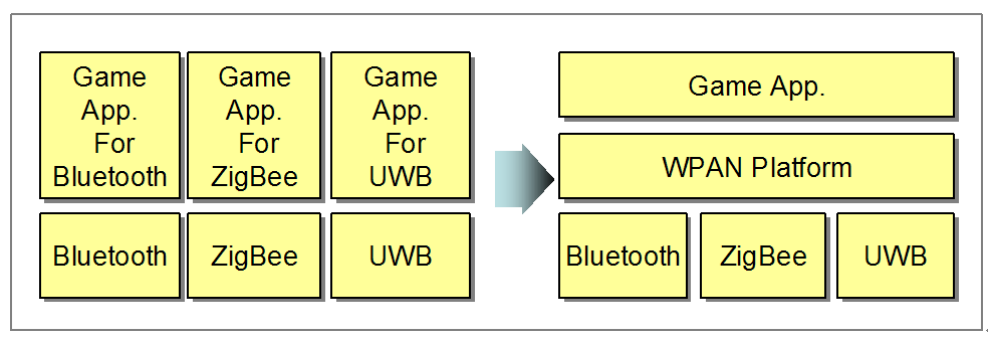

Figure 3: Platform that is independent of WPAN physical layer

In fact, the characteristics of each WPAN technology must be considered. We are currently developing a Platform Layer for ZigBee and UWB. Also, the development of an API set design for the differences between the various WPAN technologies remains future work.

\section{WPAN Connection Scheme considering user scenario}

In this section, we introduce various issues concerning the WPAN services in the handset and the WPAN connection scheme. WPAN services need a usage scenario that is different from cellular network services. This can be inconvenient for the user. The WPAN connection scheme considers these points and makes it easier for the user to enjoy the services.

\subsection{Easy-to-use WPAN connection scheme}

To make a connection between devices in the WPAN, one of the devices must be in the standby mode. However, it is inconvenient for the user to set the WPAN handset to the standby mode manually to receive the WPAN service. For example, if the user enters a zone providing for LBS (location based service) and sets the handset to the standby mode manually to receive LBS messages through the WPAN, he or she may feel that the process is complicated. This can be a drawback for such services. Hence, the user should be able to receive any WPAN event without needing to type any input into the handset.

To overcome this problem, we present a scheme which enables the new connection to be conveniently set up. Figure 4 shows the processes of the WPAN platform for the serial port channel connection.

The detailed scenario is as follows:

- The WPAN handset always opens one serial port channel to receive a WPAN event. If other devices try to connect to this WPAN handset, they must try to connect to this serial port channel.

- The connection request to the serial port arrives from the sending device to the handset.

- When receiving a WPAN event (sp_connected_event) for the connection to the open serial port channel, the PAN agent executes the WAM and sends information including the BD_ADDR of the sending device and event (sp_connected_event) to the WAM. 


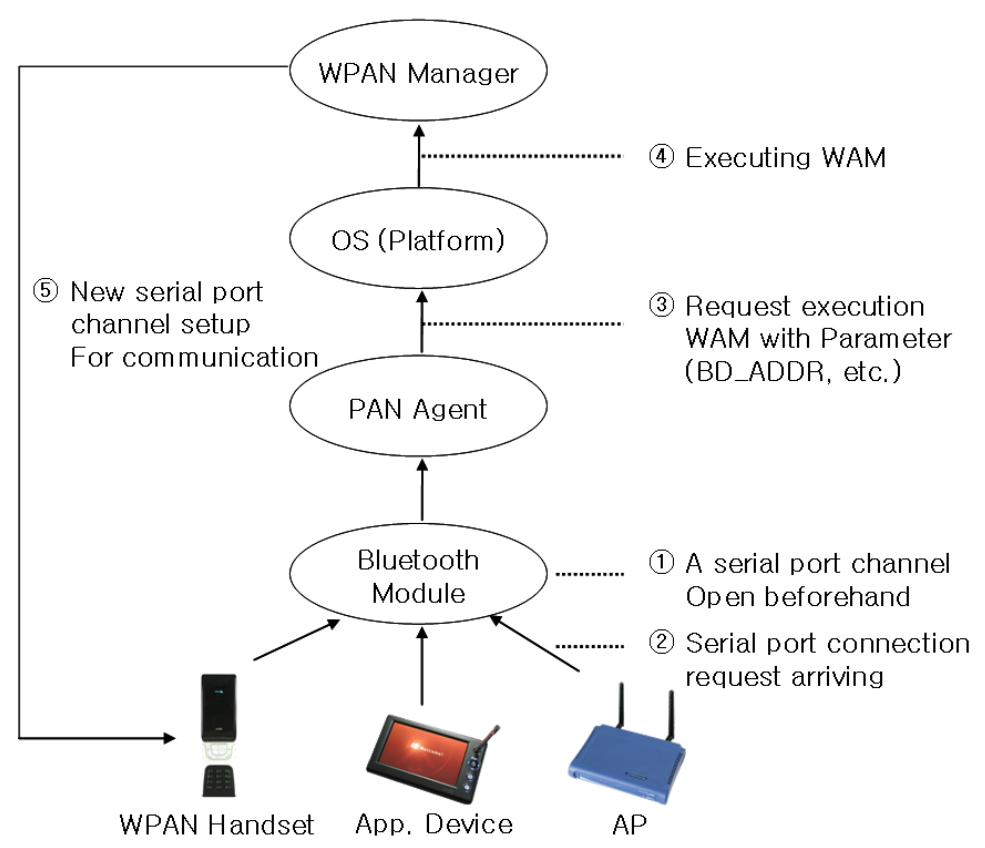

Figure 4: The process used to make a new WPAN connection

- The WAM is executed.

- The WAM creates a new SPP channel, connects to the sending device, and closes the existing connection. Then all events are processed using the new serial port channel. Also, connection requests from other more devices are handled using the conventional serial port channel.

If the event is managed at a lower level, rather than the platform level, additional services cannot be provided. Using the proposed method, however, the event is handled at the platform level and the user can be provided with additional services.

\subsection{Authentication between Devices}

The WPAN has its own authentication process according to the security level. In Bluetooth, almost all of the devices use Security Level 2 and the user must input a PIN code to the device. This can be a drawback for WPAN services.

To overcome these problems, in this paper, a pre-defined number is used as the PIN code for the connection. To compensate for the resulting lack of security, the WPAN platform authentication process is performed after the serial port channel connection is opened. We adopt the Blowfish encryption algorithm. After the connection is established, the authentication procedure using the Blowfish algorithm is performed at the serial port level and is independent of the Bluetooth encryption. After the successful authentication, the serial port channel remains open. Otherwise, it is closed. This can block anonymous connections from external devices. Figure 5 shows the authentication and execution procedures used in the proposed WPAN platform. Devices which are successfully authenticated can execute all WPAN applications of the WPAN handset. 


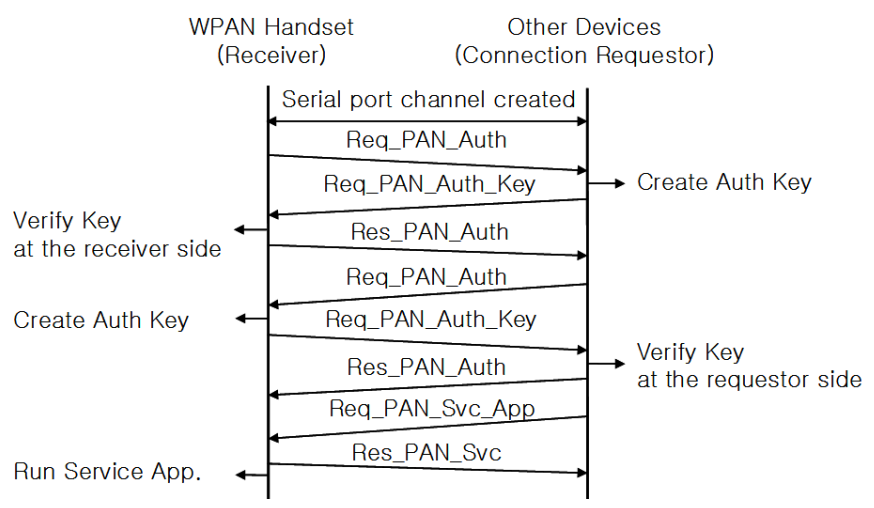

Figure 5: Authentication and application execution flow

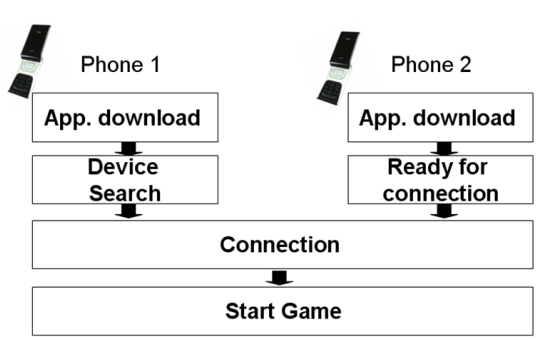

(a) Conventional WPAN user scenario

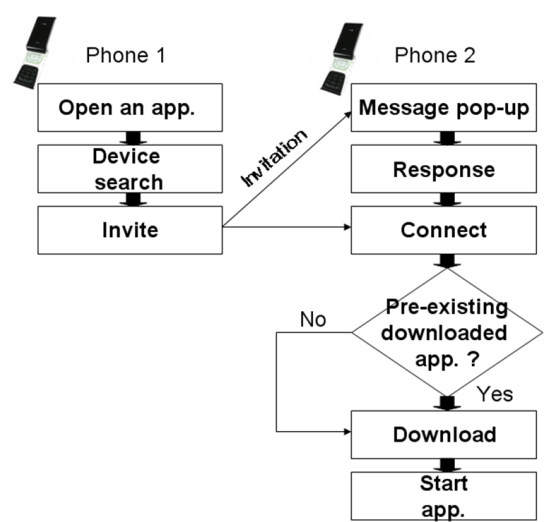

(b) The proposed user scenario for WPAN applications

Figure 6: WPAN application scenario for usability

\subsection{User scenario for the WPAN applications between users}

One of the major drawbacks of WPAN applications is the user scenario. Because there is no standard of user scenario for WPAN applications in mobile phones, the usage of applications depends on the manufacturers of the mobile phone and other WPAN devices.

In the conventional method, for phone to phone applications using WPAN, user 1 executes a WPAN application and waits for the response, as shown in Figure 6(a). Then, user 2 executes the application and connects to user 1 . The connection is established only when the users have downloaded the application. If the application has not yet been downloaded, the user must do so manually. This is inconvenient for the user and makes the usage complicated.

In the proposed method, as shown in Figure 6(b), the connection is set up even if user 2 does not have the application. In this case, user 2 can download the application through the cellular network after the connection is made.

\section{WPAN Server Platform}

To provide the user with various converged network services, the server should manage the information of the devices that are connected to the WPAN handset and handle the contents and 


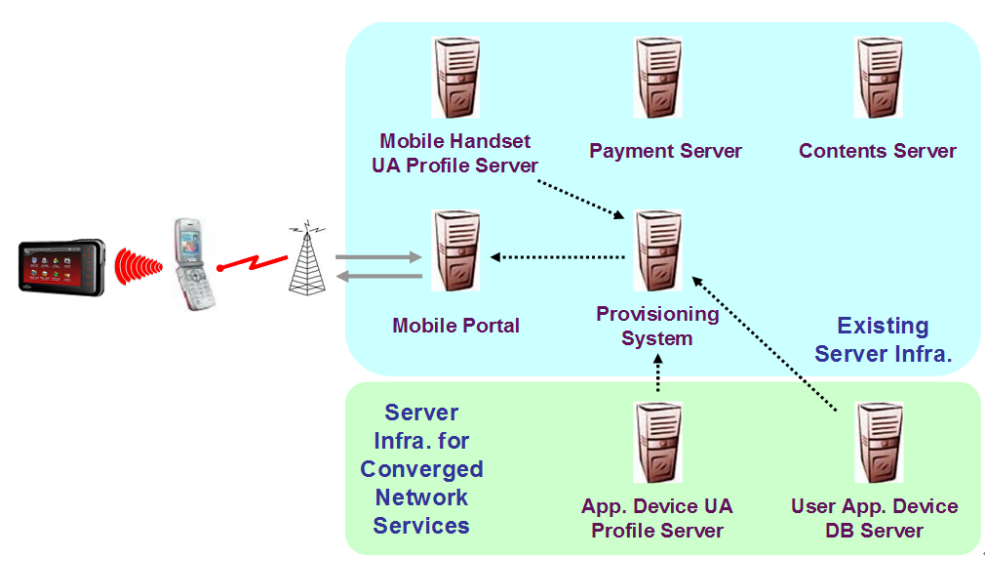

Figure 7: Server infrastructures for converged network services

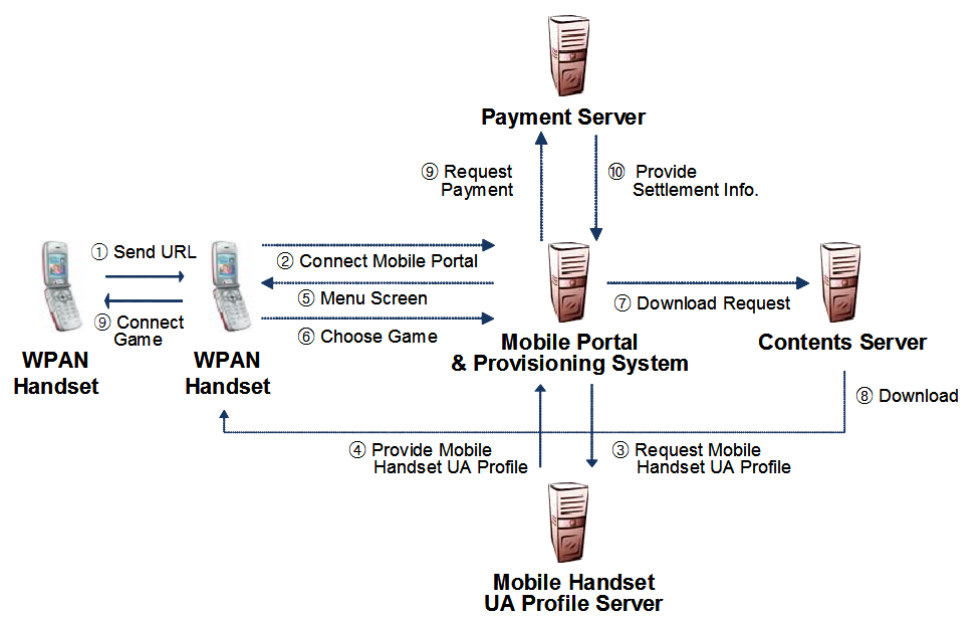

Figure 8: Server infrastructures and service flow for a converged network game

services according to the devices. We present a WPAN server architecture for WPAN applications and services, as shown in Figure 7.

Service providers keep the DB (Data Base) information of the subscriber's mobile handset in a UA (User-Agent) Profile Server. This enables the user to receive appropriate services for the user's mobile handset. Likewise, in the proposed converged network service, the service provider must keep the information of the application devices and match them to the user's mobile handset. For this purpose, the App. Device UA Profile Server and the User App. Device DB Server are required.

- The App. Device UA Profile Server provides the information required for the services according to the App. Device for the provisioning system. It manages properties such as the information of the App. Device and the service categories.

- The User App. Device DB Server handles the list of all of the App. Devices that the subscriber owns.

- When a WPAN handset connects to a Mobile Portal, the Provisioning System provides the information about mobile handset connected to the Mobile Portal using the Mobile 


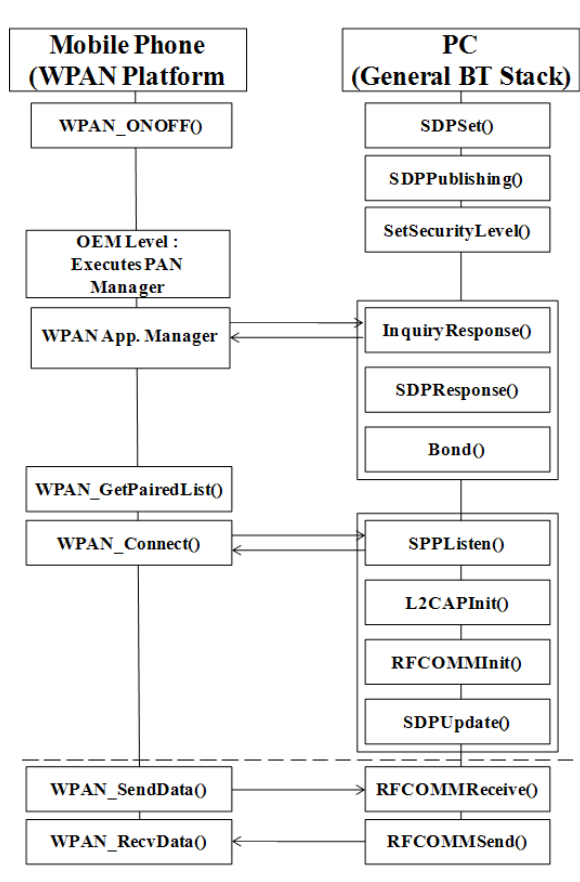

(a) WPAN platform

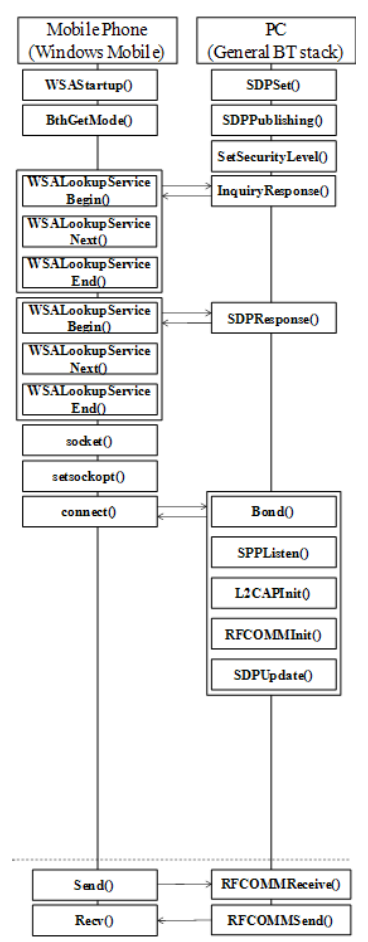

(b) MS stack

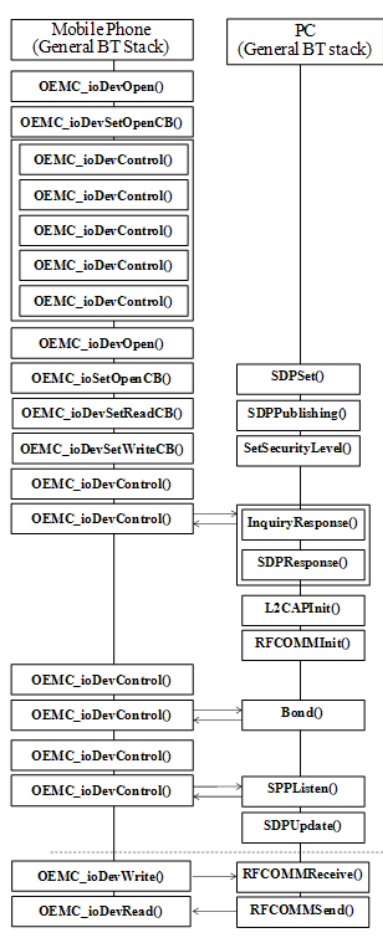

(c) General BT stack

Figure 9: API flows and number of API calls for connection using WPAN platform, general BT stack and MS stack

Handset UA Profile Server, the App. Device UA Profile Server and the User App. Device DB Server. Afterwards, the Mobile Portal can provide the user with proper contents and services. There must be an additional interface between the conventional provisioning system and the additional servers.

Figure 8 shows an example of the server infrastructure and service flows for mobile game contents. One can request to play a WPAN game with another user by transmitting the game ID and the URL location of the game. If the game does not exist in the handset, its contents can be downloaded using the URL information. In this case, the mobile portal decides whether the game is appropriate for the handset through the Mobile Handset UA Profile System. If so, the game is downloaded to the handset using both the contents server and the payment server.

For the connection of application devices such as PMP, PDA, and MP3P, we must use the App. Device UA Profile System and the User App. Device DB Server to download the contents.

\section{Performance Evaluation of the presented WPAN platform}

In this section, we present a performance evaluation of the proposed WPAN platform considering API calls for application development. In fact, performance evaluation of a platform depends on applications and performance indices. In this section, we focus on the number of API calls for an application development using WPAN core APIs in Table 3.

Although the comparison depends on applications and programming language, we make evaluation based on $\mathrm{C} / \mathrm{C}++$ APIs. We design $\mathrm{PC}$ to phone connection application for the different API sets, which are the presented APIs in Table 3, Windows Mobile Bluetooth stack and general BT stack. 


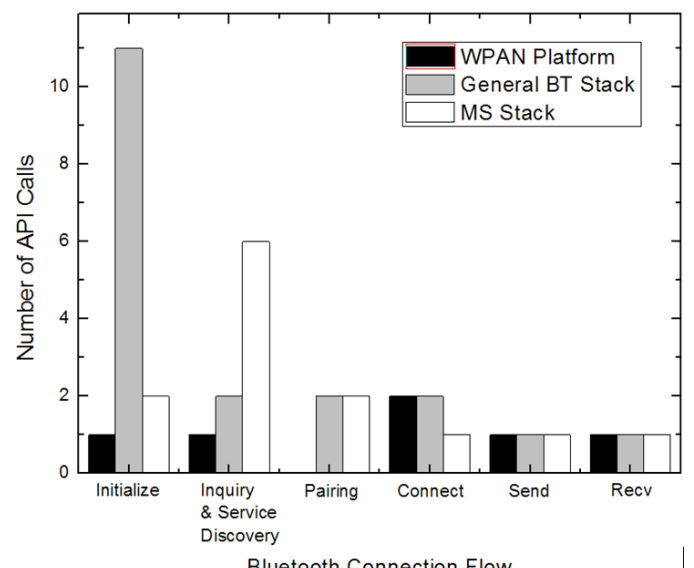

Figure 10: Number of API calls for different API sets

Table 4: Number of API calls

\begin{tabular}{|c|c|c|c|}
\hline & WPAN Platform & MS Stack & General BT Stack \\
\hline Initialize & 1 & 2 & 11 \\
\hline Inquiry and Discovery & 1 & 6 & 2 \\
\hline Pairing & 0 & 2 & 2 \\
\hline Connect & 2 & 1 & 2 \\
\hline Send & 1 & 1 & 1 \\
\hline Receive & 1 & 1 & 1 \\
\hline Total & 6 & 13 & 19 \\
\hline
\end{tabular}

Using the WPAN platform, the connection is processed as the following sequences.

- Turn on the Bluetooth module. (WPAN_ONOFF())

- Invoke the WPAN manager and search for WPAN devices. Then, exchange the PIN code and perform authentication. (WPAN Manager)

- Get the list of the searched devices. (WPAN_GetPairedList())

- Request connection and make the connection. (WPAN_Connection())

- Exchange data between PC and handset. (WPAN_SendData(), WPAN_RecvData())

Figure 9 shows the API flows for the PC to phone connection using WPAN platform, MS stack and general BT stack, respectively. As shown in Figure 9, the connection procedure can be made easily using WPAN platform.

Figure 10 and Table 4 show the number of API calls for the process of application. The total number of API calls is about 6, 13 and 19 for WPAN platform, MS stack and general Bluetooth stack respectively, in a sample application. As shown in Figure 9, Figure 10 and Table 4, we can develop the WPAN applications easily using WPAN Platform APIs. 


\section{Illustrative Services}

To show the validity of the proposed WPAN platform, we present illustrative service examples adopting the proposed WPAN platform. As examples of converged network services, a PC to phone connection, handset to handset game, Voice Terminal, PMP, and LBS are introduced. For example, aside from the existing Bluetooth handset to handset games, the proposed game design considers a full connection between the cellular network and the WPAN.

\subsection{PC to phone connection}

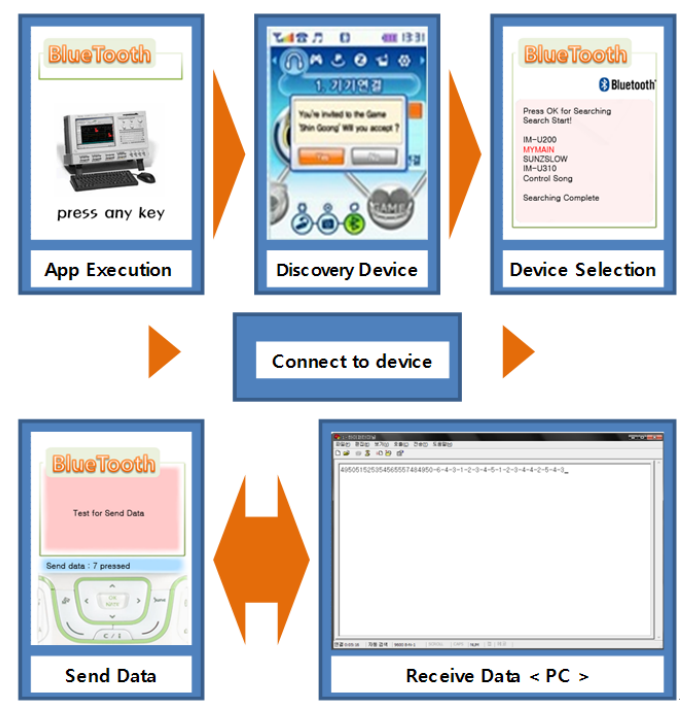

Figure 11: Example of data transmission between PC and mobile phone
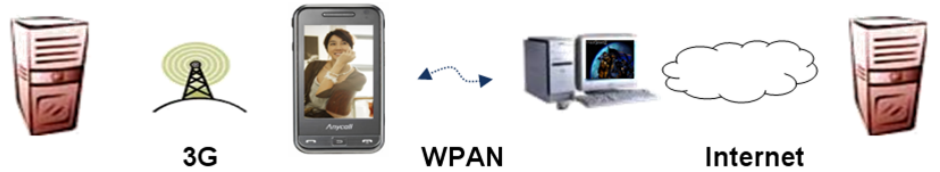

Figure 12: Converged network service using PC to phone connection

Although PC to phone connections are widely used already, we present an implementation of a $\mathrm{PC}$ to phone connection using the proposed platform in order to validate the proposed WPAN APIs for the connection to general Bluetooth devices. Using a PC, the user can download various multimedia contents, as well as phone books, pictures, etc. In Section 7, overall connection procedure is briefly introduced already. Figure 11 shows an example of data transmission between the PC and mobile phone.

Also, as seen in Figure 12, various services can be made through the converged networks, i.e., Bluetooth connection between PC and mobile phone, internet and $3 \mathrm{G}$ network. 


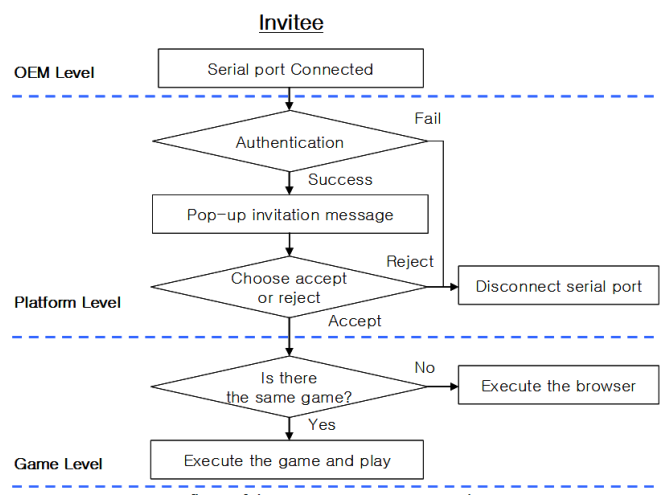

Figure 13: A flow of the WPAN game setup and termination

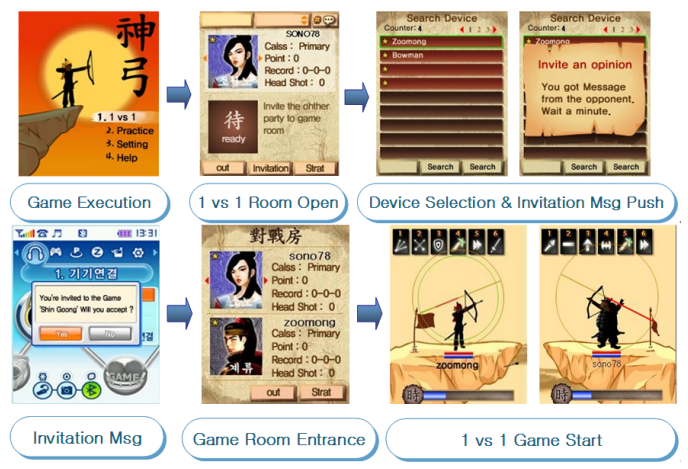

Figure 14: A WPAN game scenario with screen shots

\subsection{Mobile Game using WPAN Platform}

In a conventional mobile game in a mobile phone, people play locally after downloading the game using a cellular network. Otherwise, they can enjoy online games through a cellular network. In a converged network environment, we can provide various service models for mobile games by using both the cellular network and WPAN. First, we can apply new items or characters to the existing game through the cellular network and it is possible to download charged items with the connection of the mobile payment service. In addition, we can download the scenarios of the game itself. Second, it is possible to play the game among WPAN game groups. This can be used in a large scale game such as a role playing game.

Using the platforms and schemes presented in this paper, a new mobile game model can be derived. As shown in Figure 6 and Figure 13, we can have a new user scenario model for mobile games. A game user can invite any new user or registered user to play a WPAN game. A counterpart who receives a WPAN event for the game request can choose to accept or reject the invitation. If the game does not exist on the receiver side, the WPAN platform (WPAN App. Manager, to be exact) invokes the WAP (Wireless Application Protocol) browser in order to download the game application to the mobile handset through the cellular network. Figure 13 shows the flow of the WPAN game setup and termination in terms of the invitee and Figure 14 shows an example of the WPAN game scenario with screen shots. Likewise, the WPAN platform must identify the existence of the game according to the request message. If the game is not present on the handset, it should be downloaded and the connection setup should be processed. 


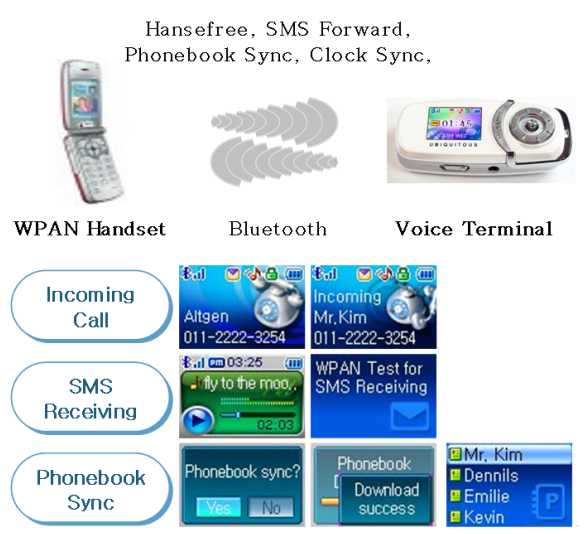

(a) Voice Terminal and its functions

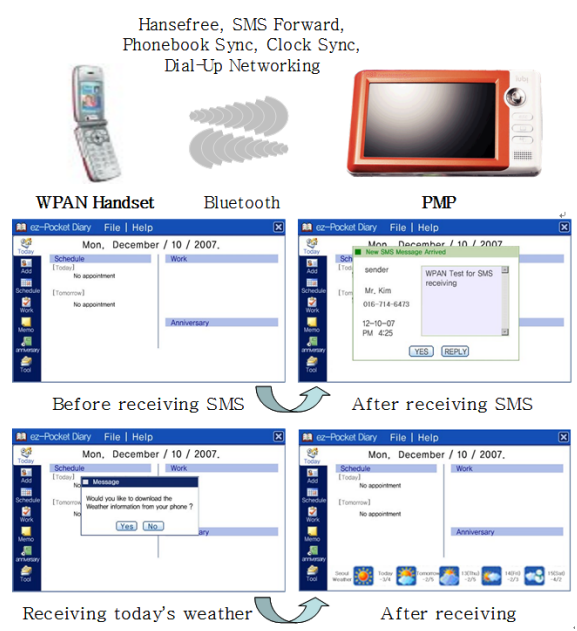

(b) PMP interacting with a WPAN handset and its functions

Figure 15: Voice Terminal and PMP

\subsection{Voice Terminal}

A Voice Terminal is a digital device using the WPAN platform functioning as both an MP3 Player and a headset. In addition, users can receive SMS messages and make calls by selecting one of the phone numbers from the downloaded phone book.

By directly using the Handsfree profile of Bluetooth in PAL APIs, one audio link (SCO Link) and one data link (ACL Link) are established between the handset and Voice Terminal. The SCO link is used for the Handsfree function and PCM data is transmitted using the SCO link. The ACL link is used for control commands (AT commands). After the Handsfree profile connection is established, another data link (ACL Link) is connected to the Voice Terminal. Through this data link, the SMS or phonebook can be downloaded from the handset. Users can see the SMS and make calls with the Voice Terminal. Likewise, new functions can be added using new data links and new protocols.

Figure 15(a) shows the appearance of the Voice Terminal and its functions.

\subsection{Portable Media Player}

A PMP can be connected to the handset and various applications can be provided. First, SMS forwarding, phonebook, clock sync, and Handsfree functions can be adapted for the PMP. Second, The PMP can be connected to the internet through the WPAN and the cellular network using dial-up networking.

Figure 15(b) shows an overview of the implemented PMP and some of its functions.

\subsection{LBS using Bluetooth AP}

We can develop Location Based Services using WPAN platform in mobile phone. In the BT based service, the location estimation can be more precise than those of GPS and cellular network for indoor applications. Figure 16 shows a basic concept of LBS using Bluetooth. 


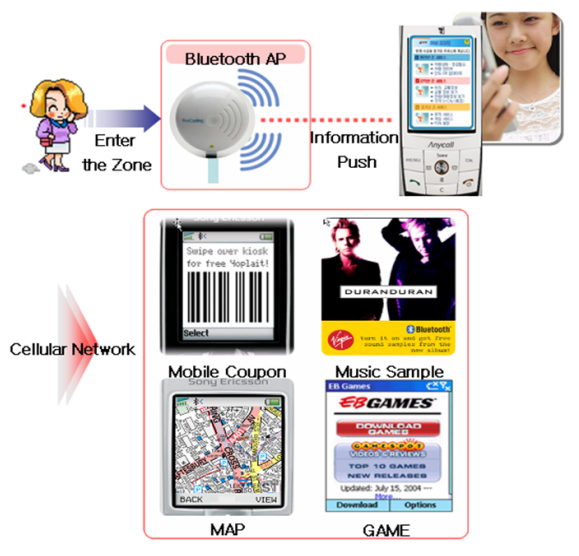

Figure 16: . Location Based Service using BT access point

\section{Conclusion}

In this paper, we proposed a WPAN platform architecture that integrates a cellular network and a WPAN, presenting illustrative service implementations of the platform. For the development of wireless communication services, we introduced the WPAN handset platform, WPAN server platform, and WPAN connection scheme.

The WPAN handset platform supplies WPAN APIs and cellular network APIs in order to integrate both networks. The WPAN APIs are abstracted for the easy development of WPAN services. Considering the various user scenarios, a WPAN connection scheme, which consists of easy-to-use WPAN connections, a WPAN authentication scheme and user scenario for WPAN applications was proposed. The WPAN server platform manages the connected devices and content related functions.

To demonstrate the applicability of the proposed platform, a PC to phone connection, mobile game, Voice Terminal, PMP and LBS implementation were discussed. The proposed architecture was implemented based on Bluetooth and has since been commercialized.

Also, as part of this study, the basic concept of converged network services was proposed and adopted as work item under the name of CPNS (Converged Personal Network Service) in OMA (Open Mobile Alliance) and AD (Architecture Document) process is in progress.

The development of an API set design for the differences between the various WPAN technologies remains future work.

\section{Acknowledgments}

This work was supported in part by the research program 2009 of SK Telecom, Korea and also supported in part by the Ministry of Knowledge Economy (MKE), Korea, under the Information Technology Research Center (ITRC) support program supervised by the National IT Industry Promotion Agency (NIPA) (NIPA-2011-C1090-1121-0005). K.-D. Chung is the corresponding author.

\section{Bibliography}

[1] Mark Weiser, The Computer for 21st Century, Scientific American, pp. 94-104, Sep., 1991. 
[2] H. Kanna, N. Wakabayashi, R. Kanazawa, and H. Ito, Home appliance control system over Bluetooth with a cellular phone, ICCE 2003, pp. 380 - 381, 2003.

[3] A. K. Salkintzis, Interworking Techniques and Architecturees for WLAN/3G Integration toward 4G Mobile Data Networks, IEEE Transactions on Wireless Communications, vol. 11, no. 3, pp. 50-61, June 2004.

[4] D. I. Axiotis, T. Al-Gizawi, K. Peppas, E. N. Protonotarios, F. I. Lazarakis, C. Papadias, and P. I. Philippopoulos, Services in interworking 3G and WLAN environments, IEEE Transactions on Wireless Communications, vol. 11, no. 5, pp. 14-20, Oct., 2004 .

[5] M. Buddhikot, G. Chandranmenon, S. J. Han, Y. W. Lee, S. Miller, and L. Salgarelli, Integration of 802.11 and third generation wirelesss data networks, Proceedings of IEEE Infocom, 2003.

[6] M. Buddhikot, G. Chandranmenon, S. J. Han, Y. W. Lee, S. Miller, and L. Salgarelli, Design and implementation of a WLAN/cdma2000 interworking architecture, IEEE Communications Magazine, vol. 41, no. 11, pp. 90-100, Nov. 2003.

[7] B. Ahlgren, L. Eggert, B. Ohlman, and A. Schielder, Ambient networks : bridging heterogeneous network domains, PIMRC 2005, pp. 937-941, Sept. 2005.

[8] I.-H. Kim, H.-J. Kim, and G.-M. Jeong, WPAN platform design in handset integrating cellular network and its application to the mobile game, Lecture Notes in Computer Science, vol. 4097, pp.103-111, 2006.

[9] J. H. Hwang, N. P. Kim, T. S. Jeong, and J. S. Koh A Framework for IM Interworking with Heterogeneous Networks, Fifth International Conference on Networking and Services, Valencia, Spain, April, pp.426-431, 2009.

[10] Specification of the Bluetooth System: Volume1, Core, Version 2.1, Bluetooth SIG, 2007

[11] ZigBee Document 053474r06 Version 1.0, ZigBee Alliance Std., 2004.

[12] WiMedia UWB PHY Layer Specification, Version 1.1, WiMedia Alliance, 2005.

[13] P. D. Garner, Mobile Bluetooth networking: technical considerations and applications, 4th International Conference on 3G Mobile Communication Technologies, pp. 274-276, 2003.

[14] JSR-82, JavaTM APIs for Bluetooth, June, 2006.

[15] S. Chetan, J. Al-Muhtadi, R. Campbell, and M. D. Mickunas, A middeleware for enabling personal ubiquitous spaces, CCNC 2005, pp. 223-228, 2005.

[16] Bluetooth Compatibility Certification program Management Document Revision 2.1, CTIA, 2009

[17] CTIA Bluetooth Compatibility Test Plan Revision 2.1, CTIA, 2009.

[18] P. Bellavista and A. Corradi, The Handbook of Mobile Middleware, Boston: Auerbach publications, 2006.

[19] 3GPP TS 22.259 V8.3.0, Technical Specification Group Service and System Aspects ; Service requirements for Personal Network Management (PNM); Stage 1, June, 2006.

[20] 3GPP TS 23.259 V1.0.0, Technical Specification Group Services and System Aspects; Personal Network Management (PNM); Procedures and Information Flows, Aug, 2007 